\title{
A NOTE ON MONOMIALS IN SEVERAL COMPLEX VARIABLES
}

\author{
G. G. WEILL
}

AbstraCt. Monomials in $C^{n}$ are characterized in the polydisk algebra $A\left(U^{n}\right)$ as functions whose modulus is constant on the distinguished boundary of $U^{n}$ and whose zero set has an intersection with the diagonal of $U^{n}$ consisting (at most) of the origin.

The note answers a question raised by $F$. Norguet in his course notes Fonctions de plusieurs variables complexes, Paris, 1971. The following characterization of monomials in the polydisk algebra is a generalization of a result of $\mathbf{R}$. Bojanic and $\mathrm{W}$. Stoll about a characterization of monomials among entire functions [1].

We use the following notations:

$U^{n}$ is the open unit polydisk in $C^{n}$.

$T^{n}$ is the distinguished boundary of $U^{n}$.

$\Delta^{n}=\left\{(\lambda, \cdots, \lambda) \in \bar{U}^{n}, \lambda \in \bar{U}^{1}\right\}$ is the diagonal of $\bar{U}^{n}$.

$A\left(U^{n}\right)$ is the algebra of functions analytic in $U^{n}$ and continuous in $\bar{U}^{n}$. For $f \in A\left(U^{n}\right)$ we call $Z_{f}$ the zero set of $f$ in $\bar{U}^{n}$. We recall that if $f, g \in A\left(U^{n}\right)$ and $f\left|T^{n}=g\right| T^{n}$ then $f=g$.

THEOREM. Assume that $f \in A\left(U^{n}\right)$ satisfies

(i) $|f| T^{n} \mid=1$,

(ii) $Z_{f} \cap \Delta^{n} \subset\{(0, \cdots, 0)\}$.

Then $f=c z_{1}^{k_{1}} \cdots z_{n}^{k_{n}}$ where $c \in T^{1}$.

ProOF. Let $z \in T^{n}$. Then $f_{z}(\lambda)=f(\lambda z)$ is a finite Blaschke product in $A\left(U^{1}\right)$ since (i) $\Rightarrow f$ is an "inner function" in $A\left(U^{n}\right)$, hence a rational function [2, p. 112]. Hence $f_{z}(\lambda)=c(z) \lambda^{p(z)}$ by (ii), where $p(z)$ is nonnegative integer valued. For $\lambda=1, f(z)=c(z) \forall z \in T^{n}$, hence $f_{z}(\lambda)=$ $f(z) \lambda^{p(z)}$. This shows that $p(z)$ is continuous integer valued on $T^{n}$ for fixed $\lambda \in \bar{U}^{1}$. Hence $p(z)=k$, a fixed nonnegative integer since $T^{n}$ is connected.

It follows that $f(\lambda z)=\lambda^{k} f(z) \forall z \in T^{n}$ hence also for $\forall z \in U^{n}$. As seen from the Taylor expansion of $f, f$ must be a homogeneous polynomial

Received by the editors March 21, 1973 and, in revised form, May 9, 1973.

AMS (MOS) subject classifications (1970). Primary 32A10, 32A15; Secondary $32 \mathrm{E} 25$.

Key words and phrases. Monomials, several complex variables, polydisk algebra. 
of degree $k$. (This part of the proof parallels an argument of S. Bochner [3].) Denote by $k_{i}$ the degree of $f$ in $z_{i}(i=1, \cdots, n)$. Write $f(z)=Q_{i}(z) z_{i}^{k_{i}}$ plus terms of lower degree in $z_{i}$ where $Q_{i} \neq 0$ is a polynomial that does not involve $z_{i}$. There is at least one point of $T^{n}$ where all $Q_{i}$ are $\neq 0$. (If some $Q_{i}$ were zero at each point of $T^{n}$, then $\prod_{i} Q_{i}$ would be zero on $T^{n}$ and hence identically zero on $U^{n}$. Hence one of the $Q_{i}$ 's would be identically zero-a contradiction.)

Assume such a point is $(1,1, \cdots, 1)$; now $f(\lambda, 1,1, \cdots, 1)$ is a Blaschke product hence it has all its $k_{1}$ zeros in $U^{1}$. An easy index computation (see [2, p. 89]) shows that $f(\lambda, \lambda, \cdots, \lambda)$ has degree $k_{1}+\cdots+k_{n}$. It follows that $f$ is a monomial, $f=c z_{1}^{k_{1}} \cdots z_{n}^{k_{n}}$ where $c \in T^{1}$.

\section{BIBLIOGRAPHY}

1. R. Bojanic and W. Stoll, A characterization of monomials, Proc. Amer. Math. Soc. 13 (1962), 115-116. MR 25 \#129.

2. W. Rudin, Function theory in polydisks, Benjamin, New York, 1969. MR 41 \#501.

3. S. Bochner, Entire functions in several variables with constant absolute values on a circular uniqueness set, Proc. Amer. Math. Soc. 13 (1962), 117-120. MR 25 \#4130.

Department of Mathematics, Polytechnic Institute of Brooklyn, Brooklyn, NEW YORK 11201 